\title{
The formation of planetary disks and winds: an ultraviolet view
}

\author{
Ana I. Gomez de Castro ${ }^{1}$ \\ aig@mat.ucm.es
}

Received __ accepted _

${ }^{1}$ Fac. de CC Matematicas, Universidad Complutense de Madrid, 28040 Madrid, Spain 


\begin{abstract}
Planetary systems are angular momentum reservoirs generated during star formation. This accretion process produces very powerful engines able to drive the optical jets and the molecular outflows. A fraction of the engine energy is released into heating thus the temperature of the engine ranges from the $3000 \mathrm{~K}$ of the inner disk material to the $10 \mathrm{MK}$ in the areas where magnetic reconnection occurs. There are important unsolved problems concerning the nature of the engine, its evolution and the impact of the engine in the chemical evolution of the inner disk. Of special relevance is the understanding of the shear layer between the stellar photosphere and the disk; this layer controls a significant fraction of the magnetic field building up and the subsequent dissipative processes ougth to be studied in the UV.

This contribution focus on describing the connections between 1 Myr old suns and the Sun and the requirements for new UV instrumentation to address their evolution during this period. Two types of observations are shown to be needed: monitoring programmes and high resolution imaging down to, at least, milliarsecond scales.
\end{abstract}

Subject headings: 


\section{Introduction}

Solar sytem progenitors, from the deeply embedded sources to the weak line T Tauri Stars (WTTSs), are sources of high energy radiation (X-ray to UV); the total energy radiated in this range goes from $\sim 0.02 \mathrm{~L}_{\odot}$ measured in the very young sources through their X-ray radiation to the $0.2 \mathrm{~L}_{\odot}$ radiated in the UV during the $\mathrm{T}$ Tauri phase (or Phase TT) (Preibish 2004, Gómez de Castro 2008). Later on, in the Weak line T Tauri

Phase the energy released drop to $\sim 10^{-3} \mathrm{~L}_{\odot}$ radiated in X-ray. Being TTSs intrinsically cool stars (with $\log T_{\text {eff }} \sim 6500-3600 \mathrm{~K}$ ), surrounded by cool accretion disks radiating at infrared wavelengths, the source of this energy must be searched in the release of magnetic energy.

It is well known that the mediation of magnetic fields in the accretion process is able to heat up the plasmas since a fraction of the gravitational energy lost during accretion is invested in field amplification and dynamo action thus, radiative loses are pushed towards the high energy range. Unfortunately, in the early phases (ages $<0.1 \mathrm{Myr}$ ) most of this radiation is reabsorbed by the dense circumstellar environment (Av>3) and only the hard X-ray radiation is able to escape from the system providing direct information on the evolution of the accretion process. After $1 \mathrm{Myr}$, extinction drops enough to make the engine accessible to UV wavelengths; current technologies allow to carry out high resolution spectroscopy in the UV range which is also extremely rich in spectral tracers so a single echelle spectra can provide information on molecular, atomic and ionized gas (from singly ionized gas to very high ionization species such as Fe XII, Fe XVIII or Fe XXI). Thus, UV spectroscopy is an extremely efficient tool to study solar system progenitors from 1 Myr on with the current technology and these application will be discussed in detail below. However, one can foresee a future when microarcseconds UV imaging will be available and studies similar to those being run on the Sun, will be feasible from 1 Myr old Suns all the 
way down into the main sequence while the young planetary disk settles down and life begins to grow. This review deals with this; with a description of our current understanding of the evolution from the T Tauri phase to the modern Sun and with a non-technologically biased ambitious view of what we could learn from challenging new UV observatories. This review has been written after the end of the 1st. conference of the network for UV astronomy held in El Escorial in May 2007 where some challenging projects for new space observatories were presented; you should find references to some of them in this text.

\section{Physics to be understood I: the gravito-magnetic engine}

During the phase TT, stars count on an energy source which is not available during the main sequence evolution: gravitational energy from the infalling material. This extra energy is released either through shocks on the stellar surface or through the gravito-magnetic interaction between the star and the disk.

Shocks release the kinetic energy of the infalling material into heating at the impact point. If matter infall occurs along the field lines, all the gravitational energy is damped into heating and the gas may reach temperatures as high as $\sim 10^{6} \mathrm{~K}$. The dominant output radiation is produced by the photoionized preshock infalling gas radiating mainly in the UV range (Gómez de Castro \& Lamzin 1999; Gullbring et al. 2000). As the density of the infalling gas column is high $\left(n_{e} \simeq 10^{9}-10^{12} \mathrm{~cm}^{-3}\right)$ the thickness of the radiating column is expected to be negligible compared with the stellar radius thus, accretion shocks are observed as hot spots on the stellar surface. As such, they are expected to produce a rotationally modulated signal that has been detected in monitoring campaigns of some stars both in optical (see i.e. Petrov et al. 2001; Bouvier et al. 2003) and UV (Gómez de Castro \& Fernández 1996; Gómez de Castro \& Franqueira 1997a). An important result of these campaigns is that only $\sim 50 \%$ of the UV continuum excess is rotationally modulated. 
Thus, a significant fraction of the UV excess is not produced by the accretion shocks even in sources where rotational modulation has been detected. However, this excess decreases as the star approaches the main sequence (see Fig. 1).

In fact, the major source of high energy radiation is the dissipation of the magnetic and mechanical energy produced by the gravito-magnetic engine. A simple analogy can be made with a self-regulated hydraulic turbine: the potential energy of the gas falling from the disk into the stellar magnetic field drives to the generation of electric currents due to the Lorentz force that, in turn, create new field components through dynamo action. There are however, a great number of uncertainties in the way the system self-regulates and also on the dependence of the engine details on initial conditions such as the effective gravity of the star, the role of stellar radiation and magnetic field on the engine performance and the role of the ionizing radiation produced by the engine on the evolution of the mass storage, the disk.

The Sun, itself, provides important clues to understand the physics of the gravitomagnetic engine and its evolution. At the base of the Sun convective layer, the tachocline marks the location of the shear layer between the rigid body rotation of the radiative core and the differentially rotating convective envelope. The tachocline is significantly prolate; it is centered at $0.69 \mathrm{R}_{\odot}$ at the equator and $0.72 \mathrm{R}_{\odot}$ at latitude $60^{\circ}$ (Basu \& Antia, 2003). The tachocline thickness is $\sim 0.04 \mathrm{R}_{\odot}$. The angular velocity profile based on helioseismic inversions shows that at a latitude of about $35^{\circ}$, the radial gradient changes sign becoming negative for latitudes $>35^{\circ}$. This latitude marks the limit of the two latitude belts where the overwhelming majority of sunspots occur. There are also some indications of the meridional flow moving equatorwards below this latitude and polewards above it (see Miesch 2005). The Solar wind is (magnetic) latitude dependent during solar minimum; above $\sim 35^{\circ}$ is fast $(1000 \mathrm{~km} / \mathrm{s})$ and thin, below is slower $(300 \mathrm{~km} / \mathrm{s})$ and denser (see Fig.2 


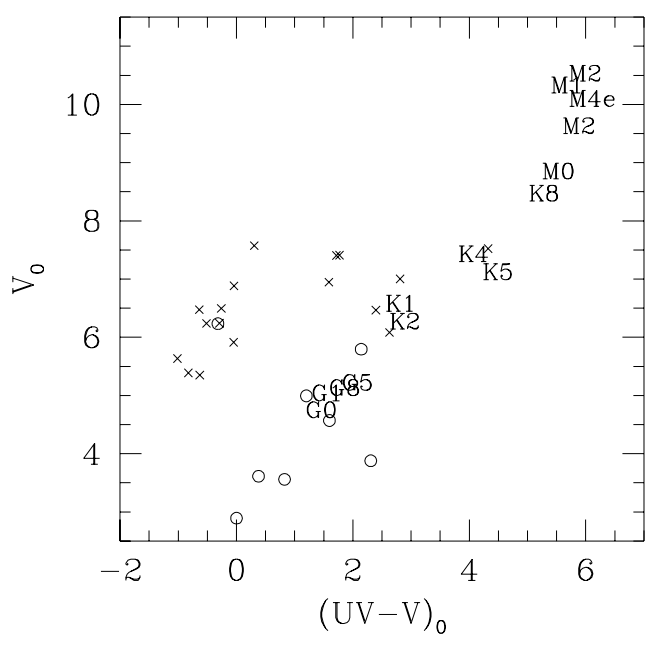

Fig. 1.- The (UV-V, V) colour - magnitude diagram for the T Tauri stars observed with the IUE satellite in the Taurus region. The crosses represent cool TTSs (spectral types later than $\sim$ K3) and the open circles warm TTSs (spectral types earlier than $\sim$ K3). The location of the main sequence is marked by the spectral types. The stars closer to the main sequence are the WTTSs (from Gómez de Castro 1997). 
from Ulysses data). The current paradigm for how solar dynamo operates includes: (1) field amplication in a turbulent downflow ( $\alpha$ effect) that is pumped downward by convection and accumulate in the overshoot region and the tachocline; (2) field amplification and organization into toroidal flux tubes and sheets by differential rotation in the tachocline; (3) magnetic instabilities (buoyancy) drives the field to the surface and (4) the Coriolis force acting on the rising structures depends on the latitude producing a latitude-dependent emergence of bipolar magnetic structures.

Going backwards in time, during the phase TT, the problem becomes complicated by the presence an additional "tachocline" or differentially rotating region attached to the convective layer. This external tachocline connects the star with the accretion disk which rotates significantly faster than the stellar surface; rotation periods during the TT phase are about $7-8$ days $\left(\Omega_{*}=0.8-0.9\right.$ day $\left.^{-1}\right)$ while the Keplerian frequency is:

$$
\Omega_{k}=11.1 \text { day }^{-1}\left(\frac{M}{M_{\text {odot }}}\right)^{1 / 2}\left(\frac{r}{3 R_{\text {odot }}}\right)^{-3}
$$

Keplerian disk corotation radius is at,

$$
r_{\mathrm{co}}=(7.2-6.9) R_{\odot}\left(\frac{M}{M_{\text {odot }}}\right)^{1 / 2}
$$

To avoid this large shear, the magnetosphere will grow to balance the toroidal component of the flux with the angular momentum of the infalling matter (Ghosh \& Lamb 1979) thus,

$$
\frac{B_{p} B_{t}}{4 \pi} 4 \pi r^{2} \Delta r \simeq \dot{M} r V_{k}
$$

where $B_{p}$ and $B_{t}$ are the poloidal and toroidal components of the field respectively, $r$ is the magnetosphere radius, $\Delta r$ is the thickness of the shear layer, $\dot{M}$ is the accretion rate and $V_{k}$ is the Keplerian velocity at the magnosphere radius. For typical T Tauri stars parameters:

$$
r_{\mathrm{mag}}=4.4 R_{\odot} \gamma^{2 / 7}\left(\frac{B_{*}}{1 k G}\right)^{4 / 7}\left(\frac{\dot{M}}{10^{-8} M_{\odot} \mathrm{yr}^{-1}}\right)^{-2 / 7}\left(\frac{M_{*}}{M_{\odot}}\right)^{-1 / 7}
$$



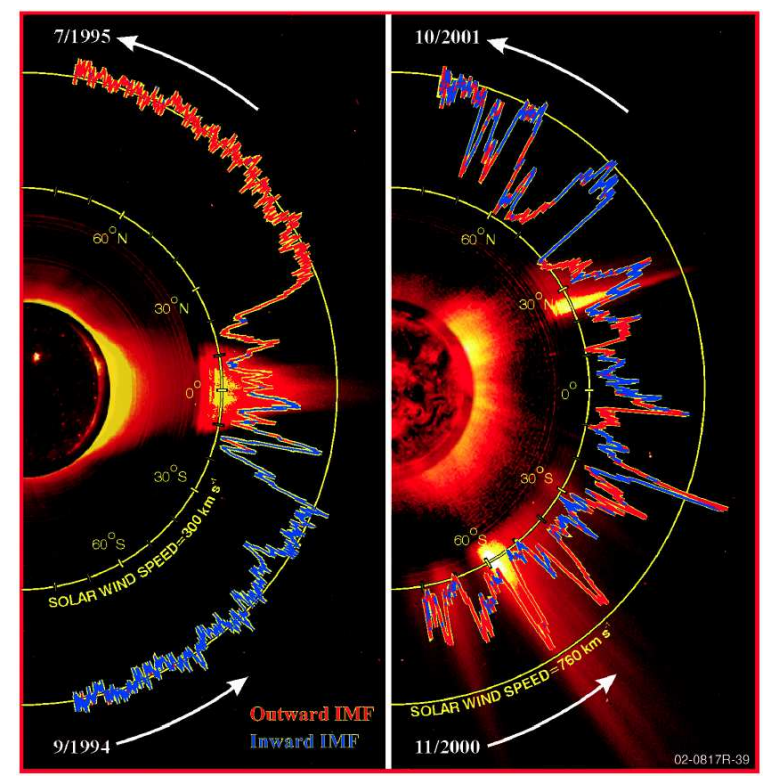

Fig. 2.- "Solar wind observations collected by the Ulysses spacecraft during two separate polar orbits of the Sun, six years apart, at nearly opposite times in the solar cycle. Near solar minimum (left) activity is focused at low altitudes, high-speed solar wind prevails, and magnetic fields are dipolar. Near solar maximum (right), the solar winds are slower and more chaotic, with fluctuating magnetic fields." (From NASA Solar Probe Web (solarprobe.gsfc.nasa.gov), courtesy of Southwest Research Institute and the Ulysses/SWOOPS team) 
where $\gamma^{2 / 7}$ is a factor about unity $\left(\gamma=\left(B_{t} / B_{p}\right)(\Delta r / r)\right.$, see Lamb, 1989). Notice that the main uncertainties in the physics, namely the ratio between the toroidal and the poloidal components and the relative thickness of the "external tachocline" are enclosed in this factor. As in the Solar interior, the shear region is fed by turbulent, magnetized material though this comes from the accretion disk instead of the convective layer. The turbulent disk dynamo is fed by the magneto-rotational instability in the acretion disk. Shear amplifies the field producing a strong toroidal component; an external dynamo sets in. This toroidal field and the associated magnetic pressure push the field lines outwards from the disk rotation axis, inflating and opening them in a butterfly-like pattern reminiscent of the helmet streamers in the solar corona, so producing a current layer between the stellar and the disk dominated regions as displayed in Fig 3. Magnetic field dissipation in the current layer produces high energy radiation and particles. The magnetic link between the star and the disk is broken and reestablished continuously by magnetic reconnection. The opening angle of the current layer, as well as its extent, depends on the stellar and disk fields, the accretion rate and the ratio between the inner disk radius and the stellar rotation frequencies. Hot, pressure driven outflows are produced from the region closer to the rotation axis while cool centrifugally driven flows are produced by the disk; plasmoids are ejected from the current layer generating a third outflowing component.

Disk-star interaction has been investigated by means of numerical simulations since the early works by Goodson et al (1997) till the last results (i.e. von Rekowski \& Brandenburg 2006). They show that the fundamental mechanism for disk winds formation is robust; numerical simulations with different parameters (disk/star fields) and initial conditions produce disk winds. Stellar winds are much more sensitive to the physical conditions and specially to the stellar field; compare the results of simulations assuming that the stellar field is a magnetic dipole (von Rekowski \& Brandenburg 2004) with those of simulations where the stellar field is prescribed through the action of the stellar dynamo (von Rekowski 


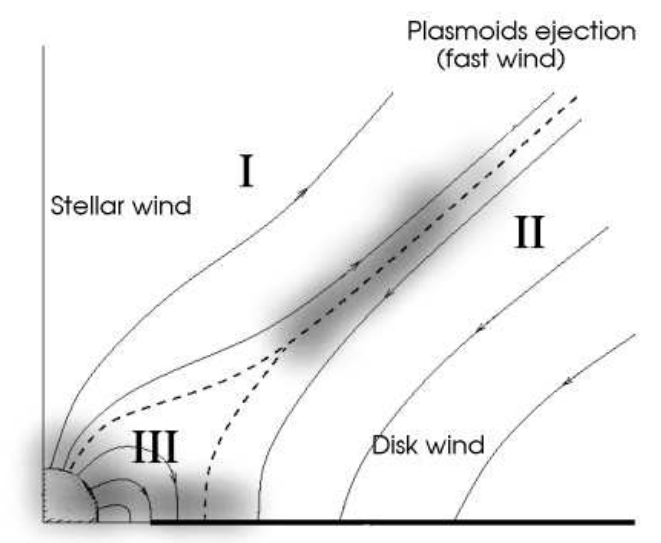

Fig. 3.- The interaction between the stellar magnetic field and the disk twists the stellar field lines due to the differential rotation. The toroidal magnetic field generated out of the poloidal flux and the associated pressure tends to push the field lines outwards, inflating them, and eventually braking the magnetic link between the star and the disk (boundary between regions I and II). Three basic regions can be defined: Region I dominated by the stellar wind, Region II dominated by the disk wind and Region III dominated by stellar magnetospheric phenomena. The dashed line traces the boundaries between this three regions. The continuous lines indicate the topology of the field and the shadowed areas represent regions where magnetic reconnection events are likely to occur, producing high energy radiation and particles (from Gómez de Castro 2004). 
\& Brandenburg, 2006). In fact, the characteristics of the accretion flow and the winds (dominant driver, temperature, terminal velocity, density, variability) depend on the physical properties of the system such as the degree of magnetization of the disk, the characteristics of the disk dynamo and the stellar field.

The bulk of the energy produced in this engine is released at UV and X-ray wavelenghts as in the Sun atmosphere. In the very early epochs, when extinction is high $\left(A_{V} \geq 3\right)$, only the X-ray radiation from the engine is detected. Later on, about 1 Myr, extinction drops and the engine can be studied in the UV. Only in the UV, the various components of the engine can be defined and studied as well as their evolution, starting during the phase TT all the way down into the main sequence.

\section{Physics to be understood II: the impact of the engine on disk evolution}

Though often neglected, the impact of the engine in the inner disk evolution is enormous. On the one hand, the engine adds a significant poloidal component in the inner disk thus favouring gas motions perpendicular to the disk as shown by the numerical simulations, on the other hand, the engine is a source of highly energetic radiation where part of the dissipation is produced at heights of some few stellar radii above the disk in the inflating current layer; this high latitude illumination favours energy absorption by the disk. Both together act to increase the disk scale height and the absorption of the radiation henceforth producing the rarification of the disk atmosphere and favoring disk evaporation close to the star. To achieve the evaporation of a standard optically thin accretion disk, the sound speed should be comparable to the keplerian velocity thus,

$$
T=\frac{G M_{*} / r_{\mathrm{mag}}}{\mu m_{H} / \gamma \kappa}=3.13 \times 10^{7} \mathrm{~K}\left(\frac{M_{*}}{M_{\odot}}\right)\left(\frac{r_{\mathrm{mag}}}{4.4 R_{\odot}}\right)^{-1}
$$


where $\mu$ is the mean molecular weight, $\gamma$ is the polytropic index and $\kappa$ is Boltzmann constant. However, this value relaxes in the presence of a poloidal field as the expected in the disk-magnetosphere interface so,

$$
T=3.13 \times 10^{7} K \frac{\beta}{1+\beta}\left(\frac{M_{*}}{M_{\odot}}\right)\left(\frac{R_{\mathrm{mag}}}{4.4 R_{\odot}}\right)^{-1}
$$

where $\beta$ is the rate between magnetic and thermal pressures. Thus, for highly magnetized environments, $T$ may drop arbitrarily. For thin accretion disk 11 penetrated by the stellar dipolar field, $B_{*}$,

$$
\begin{aligned}
\beta & =\frac{\gamma \kappa T / \mu M_{H}}{B^{2} / 4 \pi \rho} \\
& =4.77\left(\frac{M_{*}}{M_{\odot}}\right)^{7 / 8}\left(\frac{\dot{M}}{10^{-8} M_{\odot} \mathrm{yr}^{-1}}\right)^{17 / 20} \\
& \times\left(\frac{r}{4.4 R_{\odot}}\right)^{-21 / 8}\left(\frac{B_{*}}{1 k G}\right)^{-2}
\end{aligned}
$$

where $r$ is the disk radius, $B_{*}$ the stellar magnetic field and $\dot{M}$ the accretion rate. Note that $\beta$ drops to 0.02 for accretion rates of $10^{-9} \mathrm{M}_{\odot} \mathrm{yr}^{-1}$.

Another important phenomenon to be considered is that the disk is not unlocked from the engine so disk material should be subjected to the propagation of the Alfvén waves, shear waves and global alfvén oscillations driven from the interface. In summary, we might expect the inner rim of the disk to be hot with temperatures of about $10^{4} \mathrm{~K}$ well above the temperature of dust sublimation.

The role of far-UV radiation fields and high energy particles in the disk chemical equilibrium is now beginning to be understood. Bergin et al. (2003) showed how strong

\footnotetext{
${ }^{1}$ See Frank et al 2002 for the standard prescription of the thin disk density and temperature as a function the accretion rate, radius and stellar mass.
} 
Ly $\alpha$ emission may contribute to the observed enhancement of $\mathrm{CN} / \mathrm{HCN}$ in the disk. The penetration of UV photons coming from the engine in a dusty disk could produce an important change in the chemical composition of the gas allowing the growth of large organic molecules. In this context, UV photons photodissociating organic molecules at $\lambda>1500 \AA$ could play a key role in the chemistry of the inner regions of the disk, while those photodissociating $\mathrm{H}_{2}$ and $\mathrm{CO}$ will control the chemistry of the external layers of the disk directly exposed to the radiation from the central engine.

\section{Lessons learned from UV (spectroscopic) observations}

The first observations of pre-main sequence (PMS) stars in the UV were carried out with the International Ultraviolet Explorer (IUE) (1979-1997). The observations showed that pre-main sequence stars have UV fluxes exceeding those of main sequence stars by a factor of about 50. In fact, the UV excess decreases as the stars approach the main sequence as shown in Fig 1.

UV radiation provides direct information on the interaction between the star and the disk. This includes all the various components mentioned above: the shear layer, i.e.the external tachocline, the wind, the enhanced magnetospheres, mass ejections from reconnecting loops, shocks between the various wind components (among themselves and also with the disk material) and as well as the inner regions of the disk. There is a recent review on UV observations of pre-main sequence stars and young planetary disks (Gómez de Castro et al 2006) where a detailed accounting of the work carried out since the IUE times is summarized. Thus, I should concentrate on the main lessons learned from IUE and HST observations 2 that are:

\footnotetext{
${ }^{2}$ Some observations have also been obtained with FUSE but its small effective area has
} 


\subsection{About the accretion flow}

The actual measurements of infalling gas in the UV are scarce. The are hints of accretion in the large extent of the red wings of the main UV resonance lines (Herczeg et al 2005) or through the detection of redshifted absorption components on the profiles of the most prominent emission lines. However, the only target for which there is clear spectroscopic evidence of accretion shocks (in the UV) is RY Tau (see Fig. 4). Two observations of the same star obtained in 1993 and 2001 show that there is a variable redshifted component. From this single observation three important properties are learnt:

1. As described above, UV radiation from accretion shocks is predicted to be produced in the preshock region on scale heights significantly smaller than the stellar radius. Thus, it is expected that only matter falling onto the visible hemisphere can be detected at UV wavelengths. The fact that the variable flux component is redwards shifted supports these theoretical expectations. Moreover, the broadening shows that infalling matter should cover a significant fraction of the hemisphere to account for the broad distribution of projected velocities in the infalling gas.

2. The red wing extends to velocities of $250 \mathrm{~km} / \mathrm{s}$ which corresponds to the free-fall velocity 3 from $1.7 R_{*}$ which is much smaller than the fiducial values derived for the inner disk radius.

3. The UV excess is not only produced by accretion; also the wind contributes to it. Thus accretion rates derived from the UV excess, assuming that it is caused just by magnetospheric infall, are overestimated.

allowed to observed only the brightest of the TTSs and some Vega-like disks.

${ }^{3} \mathrm{RY}$ Tau mass is $1.63 \mathrm{M}_{\odot}$ and radius $2.4 \mathrm{R}_{\odot}$ according to Hartigan et al 1995 
It also adds to our understanding of magnetospheric accretion. Magnetospheric accretion was originally proposed to explain the large broadening of the TTSs H $\alpha$ lines (Muzerolle et al 1998) later detected also in the UV resonance lines of CIV, CIII (Ardila et al 2002, Herczeg et al 2005). Typical line widths are about $200-300 \mathrm{~km} / \mathrm{s}$ that exceed by far what expected from the rotation velocities of the TTSs $(10-20 \mathrm{~km} / \mathrm{s})$ even if the corotating magnetosphere is postulated to extend to some 4-5 stellar radii. Infall adds a radial velocity component to rotation of the radiating gas. As free fall velocity is:

$$
v_{f f} \simeq 315 \mathrm{kms}^{-1}\left(\frac{M_{*}}{M_{\odot}}\right)^{1 / 2}\left(\frac{R_{*}}{R_{\odot}}\right)^{-1 / 2}
$$

The observed profiles broadenings can be reproduced without difficulty. The observed broadening of $\mathrm{H} \alpha$ or $\mathrm{Mg}$ II lines do not vary significantly requiring that, at least, the spatial average of the accretion flow is rather stable. Strong variations in the accretion flow, like the reported from RY Tau UV observations, should also show in the large scale magnetosphere tracers (i.e. $\mathrm{H} \alpha$ or $\mathrm{Mg}$ II profiles).

\subsection{About the wind}

There is another possibility to broaden the line profiles: adding a radial velocity component associated with the outflow. The presence of magnetic fields and the relevance of centrifugal launching drives to the formulation of the velocity field in the wind by means of three components: axial component (along the rotation axis), radial expansion from the axis and the azimutal toroidal component (rotation around the axis); in figure 5 there is a representation of the three components for a warm centrifugal wind model (from Gómez de Castro \& Ferro-Fontán 2005). A rapid radial expansion close to the star (to guarantee that the wind density and temperature are about the observed $\mathrm{n}_{e}=10^{10} \mathrm{~cm}^{-3}$ and $\mathrm{T}_{e}=20-30 \times 10^{3} \mathrm{~K}$ values) could produce similar effects in the profiles than those predicted by magnetospheric infall. Several attempts have been made to reproduce the 


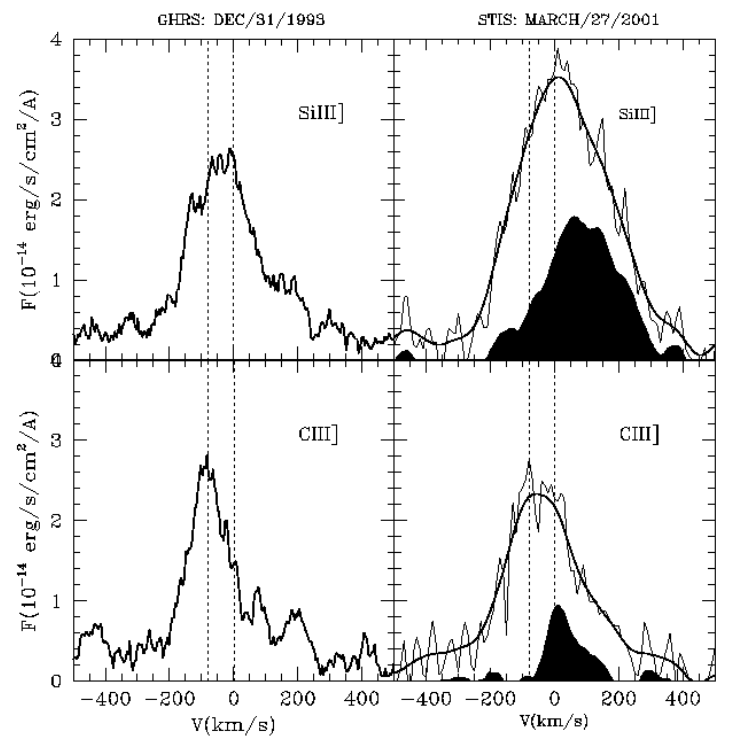

Fig. 4.- SiIII] and CIII] UV lines observed in RY Tau (from Gómez de Castro \& Verdugo 2007); RSDP processed data are plotted with a thin line and the 3-pixels average profile with a thick line. The rest wavelength of the lines and the velocity of the unresolved jet at $\simeq-80 \mathrm{~km} / \mathrm{s}$ (from Gómez de Castro \& Verdugo 2001 and Hamann 1994) are marked with dashed lines. Left panel: Observations obtained in Dec. 31st, 1993 with the GHRS. Right panel: Observations obtained in March 27th, 2001. Both lines show an excess of flux in the red wing compared with the 1993 observations; this excess is shaded in the figure. 
wind profiles with cold winds from accretion disks (Ferro-Fontán \& Gómez de Castro 2003), warm disk winds (Gómez de Castro \& Ferro-Fontán, 2005), coronal winds from accretion disks (Ferreira \& Casse, 2004) and winds driven by the star-disk interaction (Gómez de Castro \& von Rekoswki, 2008).

Cold disk winds fail to reproduce the high temperatures observed. The original wind temperature is as low as the disk one and heating has to be extracted from photoionization by the central source. However, this radiation is able to heat the gas only to mild temperatures of about $10^{4} \mathrm{~K}$. Warm disk winds produce profiles that are too narrow to reproduce all the observations; this is because the vertical thermal pressure push forces the growth of the radial velocity component to heights were the plasma is already too cool. Finally, winds driven from the star-disk interaction also produce narrower profiles than the observed in some sources as shown in Fig.6. According their UV forbidden lines profiles, TTSs can be classified in two groups: stars with broadenings with full width half maximum about $150 \mathrm{~km} \mathrm{~s}^{-1}$ that can be adjusted with the current models and stars with extremely broad profiles $\left(>250 \mathrm{~km} \mathrm{~s}^{-1}\right)$. The source of the very large broadenings have to be seek in other structures such as ion belts or plasma rings as the resolved in RW Aur (see Sect. 4.3).

Finally, UV observations have clearly proved that:

1. Warm winds are latitude dependent on scales comparable to the stellar radius. As an example, the Mg II resonance doublet has been observed in a broad sample of 17 TTSs (adding IUE and HST samples); this is the largest sample of TTSs observed in a single UV spectral line. These lines can be generically described as broad, asymmetric emission lines with typical full widths at $10 \%$ intensity of few hundreds $\mathrm{km} / \mathrm{s}$. The broad blueward shifted absorption component characteristic of mass-loss was detected in few sources (Penston \& Lago, 1983, Imhoff \& Appenzeller, 1989) but not in all of them; the degree of absorption (the asymmetry of the line) varies from not absorption 

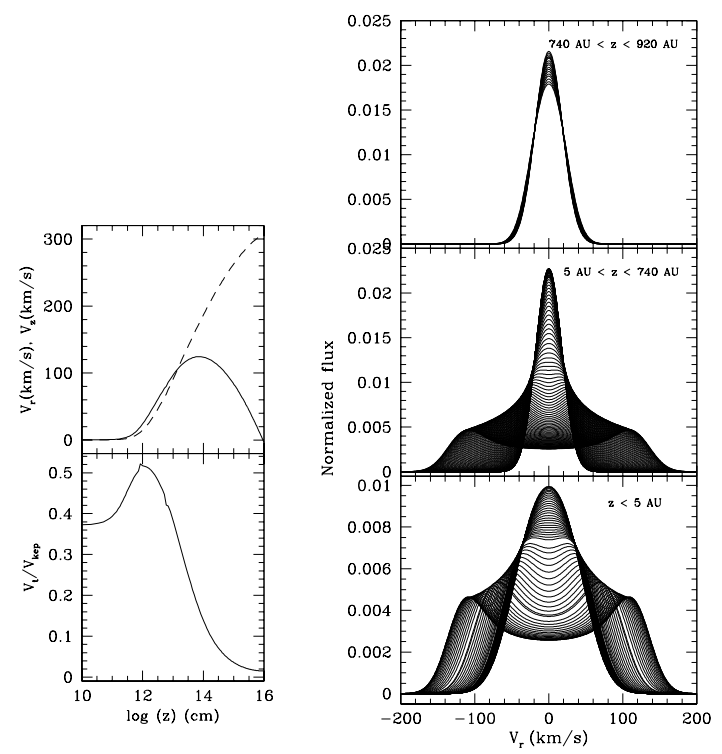

Fig. 5.- The basic kinematics of MHD centrifugal winds is outlined from the simple semiempirical model of centrifugally driven MHD winds with thermal pressure of Gómez de Castro \& Ferro-Fontán (2005). Left: Velocity field in the flow. $V_{r}$ (solid) and $V_{z}$ (dashed) are the components of the velocity along the $r$ and $z$ axes, respectively. The toroidal component of the velocity, $V_{t}$ is scaled with respect to the keplerian velocity of the disk at the radius from which the wind is ejected. Right: Line profiles generated by the wind in ring of gas at different heights along the z-axis. 

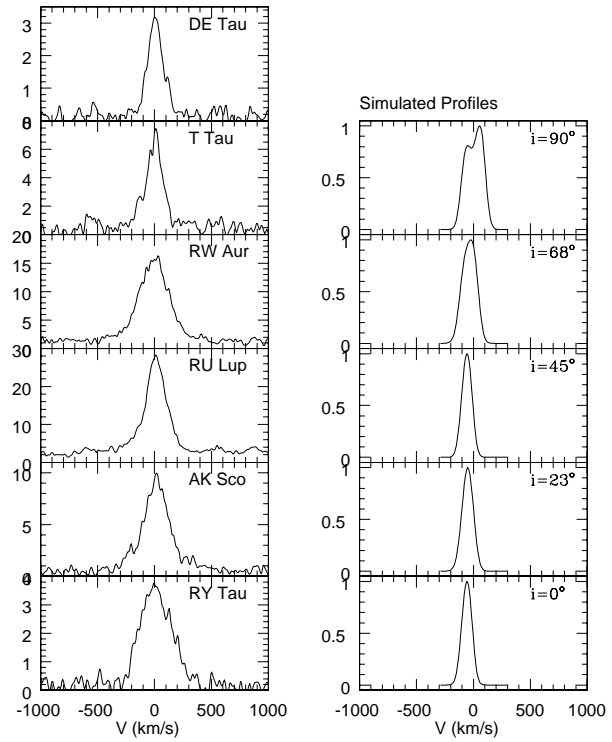

Fig. 6.- Left: Si III] profiles of the TTSs observed with HST; notice the very different line broadenings. Right: Predicted Si III] profiles for winds generated by means of the interaction between a stellar magnetosphere with stellar field $1 \mathrm{kG}$ and an accretion disk undergoing $\alpha^{2} \Omega$-dynamo effect (e.g. Krause \& Raedler, 1980) from Gómez de Castro \& von Rekowski (2008). 
to full absorption of the bluewards shifted emission (see Gómez de Castro, 1997).

2. The collimated flow, the jet, radiates in the UV as well as the bow-shock and the Herbig-Haro objects. Basically all data have been obtained with the IUE satellite (see Gómez de Castro \& Robles 2001 for a compilation). Recent observations obtained with Hopkins Ultraviolet Telescope (HUT) have shown that it is still unclear how line radiation is excited, at least, in $\mathrm{HH} 2$; in particular $\mathrm{O}$ VI is not detected as was expected in high excitation Herbig-Haro objects where line radiation is predicted to be produced in strong radiative shocks where the shock kinetic energy is damped into heating (Raymond et al 1997).

\subsection{About the inner disk and ion belts}

Strong continuum FUV emission (1300-1700 A) has been detected recently from some stars with bright molecular disks including GM Aur, DM Tau, and LkCa 15, together with inner disk gaps of few AUs (Bergin et al. 2004). This emission is likely due to energetic photoelectrons mixed into the molecular layer that likely indicates the existence of a very hot component in the inner disk.

High-resolution HST/STIS spectra have revealed, for the first time, the rich UV molecular emission in CTTSs. $\mathrm{H}_{2}$ fluorescence emission has now been studied in detail in the nearest CTTS, TW Hya, and the richness of the spectrum is overwhelming: Herczeg et

al. (2002) detected 146 Lyman-band $\mathrm{H}_{2}$ lines. The observed emission is likely produced in the inner accretion disk, as are the infrared $\mathrm{CO}$ and $\mathrm{H}_{2} \mathrm{O}$ lines. From these UV data, Herczeg et al. (2004) estimated that the warm disk surface has a column density of $N_{H_{2}}=3.2 \times 10^{18} \mathrm{~cm}^{-2}$, temperature of $T=2500 \mathrm{~K}$, and filling factor of $\mathrm{H}_{2}$ as seen from the source of the Ly $\alpha$ emission of $0.25 \pm 0.08$. The observed spectrum shows that some 
ground electronic state $\mathrm{H}_{2}$ levels with excitation energies as large as $3.8 \mathrm{eV}$ are pumped by

Ly $\alpha$. These highly excited levels may be formed by dissociative recombination of $\mathrm{H}_{3}^{+}$, which in turn may be formed by reactions involving X-rays and UV photons from the star. Also DF Tau and V836 Tau $\mathrm{H}_{2}$ emission seems to arise from the disk (Herczeg et al 2006).

In addition to this molecular component, there is increasing evidence of the existence of ion belts/rings around some TTSs. An ion belt has been detected around the TTS, RW Aur (Gómez de Castro \& Verdugo, 2003). A corotation radius of $4.4 R_{*}$ is derived and $\mathrm{a} \log T_{e}(K) \simeq 4.7$ and $\log n_{e}\left(\mathrm{~cm}^{-3}\right)=11.6$ are estimated. This was the first detection of such an structure around a classical TTS. In addition, there are indications of a similar structure around AB Dor, a weak line TTS (see Fig. 7). The structure is resolved, as in RW Aur, because there is an inner hole that allows separating the stellar/wind contribution from the belt. However in a 5.7 hours time lapse the double peaked profile is lost, and the inner part of the profile is filled in again with emission (Gómez de Castro 2002) .

\subsection{About the interaction between the disk and the wind}

AB Dor, a very bright nearby 30 Myr old star, is the only young star that has been well monitored in the UV for flares. Nine events were detected during 10.63 hours of monitoring with HST/GHRS!. The C IV and Si IV UV line profiles produced by most of the events are narrow and redshifted, indicating hot gas falling onto the star during the flare. However, the strongest event produced a very broad profile with narrow absorption slightly blueshifted. This profile lasted a few kiloseconds and thus the broad wings are most likely tracing the front shock of a CIR (Gómez de Castro 2002). In the solar system, there are three very different types of "flares", which are sudden increases of the high energy radiation and particles flux: magnetic flares (magnetic reconnection events), corotating interaction regions or CIRs (shock fronts formed by the interaction between the slow and the fast component 
of the solar wind), and coronal mass ejections. This classification also applies to TTSs and their circumstellar environments. High-resolution UV spectroscopic monitoring is required to disentangle the possible mechanisms for flares in proto-stellar systems and to study their impact in young planetary disks evolution as well as on planetary atmospheres embryos.

\section{Summary: the key observables}

In brief, the radiation produced by the accretion engine (including magnetospheres, outflows, accretion, inner disk and shock between winds and young planetary disks) is produced in the UV. To separate the various contributions is necessary either very high spatial resolution or moderate time resolution.

Current surveys show that although there are some few nearby TTSs and WTTSs sparsely distributed around the Sun (AB Dor at 14.9 pc or TW Hya at 56 pc) the nearest star forming complexes are concentrated in a Star Formation Belt (SFB) at 140 pc around the Sun which includes Taurus, Auriga-Perseus, Ophiuchus, Lupus and Chamaleon molecular clouds and several thousands of pre-main sequence stars forming in various environments (clustered as in Ophichus, sparse as in Taurus). Resolving spatial scales of a tenth of the solar radius in the SFB would allow to study the connection between the star and the outflow in full detail. For this purposes spatial resolutions of 3.3 micro arcseconds are required; thus, for a fiducial wavelength of $1500 \AA$ the aperture must be about $10 \mathrm{~km}$. Such a long baseline interferometry should be carried out in the space and the Stellar Imager project (Carpenter et al 2008) represents a first atempt to such an ambitious project.

However, the requirements are not so strong to resolve the inner disk structure, the disk wind and the plasmoids ejection from the current layer between the stellar and the disk wind. In such a case, spatial resolutions of 1-0.5 milliarcseconds (mas) would be enough to 
map the SFB sources thus requiring apertures of $20 \mathrm{~m}$ and effective areas about $10^{4}$ times those of HST/STIS; research in new coatings and detectors in the UV field (Kappelmann \& Barnstedt, 2007) as well as a clever optical design may account for a factor of ten but, still, a larger, $\sim 30 \mathrm{~m}$ aperture will be required to get $\mathrm{SNR} \simeq 10$ in the $\mathrm{C}$ IV line in reasonable exposure times (few hours). There is an ongoing project that satisfies these requirements, the Fresnel Interferometer (see Koechlin et al 2008) and some projections on the expected performance of the interferometer on the mapping of the engine are plotted in Fig. 9.

Both space interferometer projects are under study by their national space agencies and, in case they succeed, they will be available about 2030. Is there anything else to be done in the meantime?. The answer is definitely positive, time mapping will allow us to resolve the structures since the variability time scales are not the same for all the phenomena and they do not produce the same inprint in the spectra (neither in temperatures, densities or velocities). Some examples of the power of this technique have already been shown in this contribution.

High resolution spectroscopy $(\mathrm{R} \sim 50,000)$ is enough to discriminate among the various components thus, scaling with the fluxes of the weakest $\mathrm{H}_{2}$ lines (from Heczeg et al 2002) detected with the HST/STIS, a factor of 10 increase of the effective area with respect to HST/STIS is required to reach most of the sources in the SFB. The COS instrument in

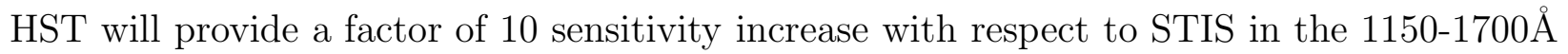
range because of its optimized optical design (see Froening et al 2008), unfortunately the orbital constrains of HST do not favour monitoring programs. High orbit missions alike the WSO-UV (see Shustov et al 2008) are better suited for this purpose.

An additional factor of 10 would be required to obtain SNR 10 in exposure times of few minutes; this short time scales are necessary to map variations in flare time scales as shown in Fig. 8 for the pre-main sequence stars in the SFB. Unfortunately, spectroscopic 
monitorings of the flaring activity in the SFB will have to wait for future missions, with collecting areas about 8-10 m, preferably located at the L2.

This work has been supported by the Ministry of Education of Spain through grant AYA2007-67726 and the Comunidad Autónoma de Madrid through grant CAM-S0505/ESP/0237 


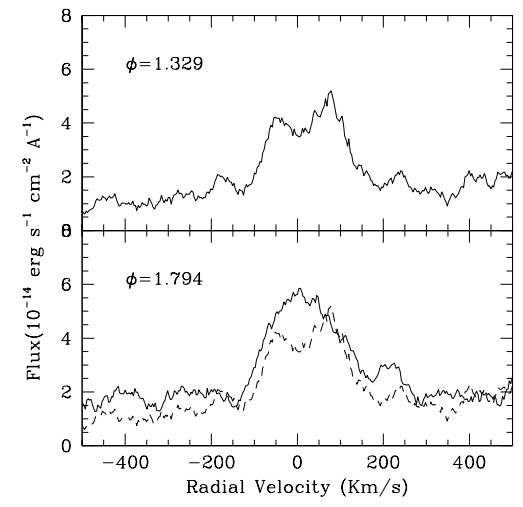

Fig. 7.- SiIII] profiles of AB Dor obtained with the HST/GHRS (see Gómez de Castro 2002 for more details). In the bottom panel, the profile at phase $(\phi) 0.329$ is overplotted (dashed line) on the profiles at $\phi=0.794$ (continuous line), for comparison. 


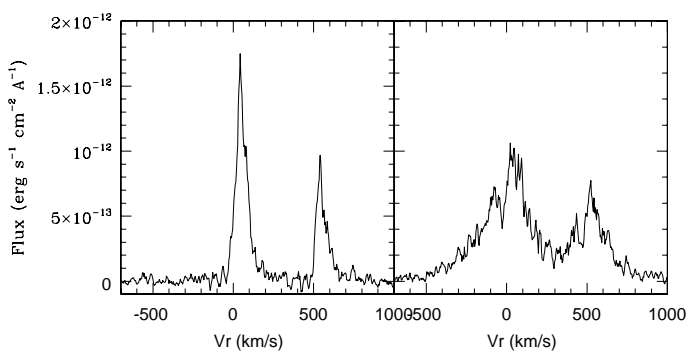

Fig. 8. - The C IV $1548 \AA$ profile of AB Dor during a normal stellar flare (left) and a transient feature probably associated with a CIR (right). Both events lasted several kiloseconds. The left profile is typical of three events that occured during the short monitoring time, while the profile on the right was observed only once. Note the presence of a narrow absorption and the very broad line wings in the right panel profile (see Gómez de Castro 2002 for more details). 

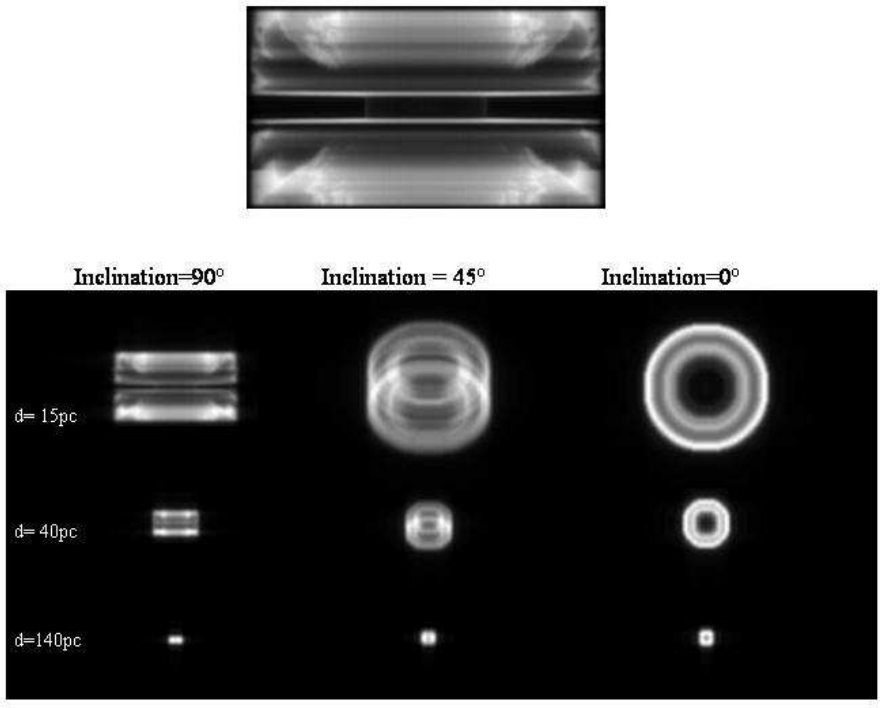

Fig. 9.- Theoretical prediction of the Si III] emissivity from numerical simulations of star-disk interaction (Gómez de Castro \& Rekowski, 2008). The stellar magnetosphere is assumed to be dipolar with a field strength at the surface of 1kG. The magnetosphere interacts with the disk which is under a moderate $\alpha$-dynamo effect 4 . The inner disk wind is magnetocentrifugally accelerated (top panel).

The convolution of the theoretical prediction with the point spread function of the 30m Fresnel Interferometer FII has been carried out by Laurent Koechlin and Truswin Raksasataya. The convolution is shown for three inclinations $\left(0^{\circ}, 45^{\circ}\right.$ and $\left.90^{\circ}\right)$ and three distances to the Earth (15 pc, $40 \mathrm{pc}$ and $140 \mathrm{pc})$. Notice that the inner ring is resolved even for $140 \mathrm{pc}$. 


\section{REFERENCES}

Ardila, D.R., Basri, G., Walter, F. M., Valenti, J. A., Johns-Krull, C. M., Astrophysical Journal, 597, 1013, 2002

Basu, S., Antia, H. M., Astrophysical Journal, 585, 553, 2003

Bergin, E., Calvet, N., D’Alessio, P., Herczeg, G. J., Astrophysical Journal, 591, L159, 2003

Bergin, E., Calvet, N., Sitko, M. L., Abgrall, H., D’Alessio, P. et al, Astrophysical Journal, 614, L133, 2004

Bouvier, J., Grankin, K. N., Alencar, S. H. P., Dougados, C., Fernández, M. et al, Astronomy and Astrophysics, 409, 169, 2003

Carpenter, K. et al, Astrophysics and Space Science, in press

Ferreira, J., Casse, F., Astrophysics and Space Science, 292, 479, 2004

Ferro-Fonán, C., and Gomez de Castro, A.I. Monthly Notices of the R.A.S., 342, 427, 2003

Froening et al, Astrophysics and Space Science, in press

Gomez de Castro, A.I. and Fernandez, M., Monthly Notices of the R.A.S., 283,55, 1996

Gomez de Castro, A.I. and Franqueira, M., IUE-ULDA Access Guide No. 8: T Tauri Stars, ESA Scientific Publication, ESA-SP 1205, 1997a

Gomez de Castro, A.I. and Franqueira, M., Astrophysical Journal, 482, 465, 1997b

Gomez de Castro, A.I. and Robles, A., INES Access Guide No. 1: Herbig-Haro Objects, ESA Scientific Publication, ESA-SP 1237, 1999

Gomez de Castro, A.I. and Lamzin,S., Monthly Notices of the R.A.S., 304, L41, 1999

Gomez de Castro, A.I. and Verdugo, E., Astrophysical Journal, 597,443, 2003a

Gomez de Castro, A.I. and Ferro-Fontán, C. Monthly Notices of the R.A.S., 362, 569, 2005

Gomez de Castro, A.I. and von Rekowski, B. Monthly Notices of the R.A.S., submitted 
Gómez de Castro, A.I., Ultraviolet Astrophysics beyond the IUE final Archive, ESA-SP 413, Nordwijk, The Netherlands, 1998

Gomez de Castro, A.I. Monthly Notices of the R.A.S., 332, 409, 2002

Gómez de Castro, A.I., 2008, Astrophysical Journal, submitted.

Goodson, A. P., Winglee, R. M., Boehm, K-H, Astrophysical Journal,489, 199, 1997

Gullbring, E., Calvet, N., Muzerolle, J. and Hartmann, L., The Astrophysical Journal, 544, 927,2000

Kappelmann, N., Barnstedt, J., Astrophysics and Space Science, 303, 147, 2006

Koechlin, L. et al., Astrophysics and Space Science, in press

Herczeg, G. J., Walter, F. M., Linsky, J. L., Gahm, G. F., Ardila, D.R. et al., Astronomical Journal, 129, 1277, 2005

Herczeg, G.J., Linsky, J.L., Walter, F. M., Gahm, G.F., Johns-Krull, C. M., Astrophysical Jourmal Supp., 165, 256, 2006

Imhoff, C.L., Appenzeller, I., Exploring the Universe with the IUE satellite, Ed. Y. Kondo, Reidel Pub. Co., 295, 1989

Lamzin, S.A., Astronomy Reports, 42, 322, 1998

Miesch, M.S., Living Reviews in Solar Physics, Max Planck Institute for Solar System Research, Germany (solarphysics.livingreviews.org) 2005

Muzerolle, J., Calvet, N., Hartmann, L., 1998, Astrophysical Journal, 492, 743

Penston, M.V., Lago M.T.V.T., Monthly Notices of the R.A.S, 202, 77, 1983

Petrov, P. P., Gahm, G. F., Gameiro, J. F., Duemmler, R., Ilyin, I. V. et al, Astronomy and Astrophysics, 369, 993, 2001.

Preibish, T., Astrophysics and Space Science, 292, 457, 2004 
Raymond, J.C., Blair, W.P., Long, K.S., Astrophysical Journal, 489, 314, 1997.

Shustov, B. et al, Astrophysics and Space Science, in press

von Rekowski, B., Brandenburg, A., Astronomy \& Astrophysics, 420, 17, 2004

von Rekowski, B., Brandenburg, A., Astronomische Nachrichten, 327, 53 